\title{
A cherry tree ripe for picking: The relationship between the acute:chronic workload ratio and health problems
}

Torstein Dalen-Lorentsen ${ }^{1}$, Thor Einar Andersen ${ }^{1}$, John Bjørneboe ${ }^{1}$, Markus Vagle ${ }^{1,2}$, Kevin Nordanger Martin ${ }^{1}$, Michael Kleppen ${ }^{1}$, Morten Wang Fagerland ${ }^{1,3}$, Benjamin Clarsen ${ }^{1,4}$

${ }^{1}$ Oslo Sports Trauma Research Center, Department of Sports Medicine, Norwegian School of Sports Sciences, Oslo, Norway

${ }^{2}$ Department of Sports, Physical Education and Outdoor Studies, University of South-Eastern Norway Horten, Norway

3 Oslo Centre for Biostatistics and Epidemiology, Research Support Services, Oslo University Hospital, Oslo, Norway

4 Department of Health Promotion, Norwegian Institute of Public Health, Bergen, Norway

\begin{abstract}
Background The acute chronic workload ratio (ACWR) is widely used to evaluate the relationship between training load and health problems. However, both ACWR and health problems can be defined in many ways, and how these methodological choices affect the relationship is unclear. Aim To investigate whether different results emerge when different definitions of ACWR and health problems are used. Methods An online questionnaire was used to collect daily health and training information from 86 elite youth footballers for 105 days. The relationship between players' training load and health was analysed using a range of different definitions of ACWR and health problem. We used a 21-day and 28-day chronic period, coupled and uncoupled calculations, and exponentially weighted moving average (EWMA) and rolling average (RA). ACWR data were categorized into three bins (low, medium and high) using pre-defined categories and z-scores, and we compared medium to high, medium to low and low to high categories. The outcome was defined in three ways: "all health problems", "all injuries", and "new non-contact injuries". We performed a total of 108 separate random-effects logistic regression analyses. Results We recorded 6250 training days and 196 health problems. Of the 108 analyses performed, 21 (19\%) identified a statistically significant $(\mathrm{p}<0.05)$ association between ACWR and health problems. A greater proportion of associations were identified when using an "all health problems" definition (33\% of analyses), when comparing low and high categories (31\%), and using EWMA (28\%).
\end{abstract}

Conclusions The relationship between ACWR and health problems was highly dependent on methodological approach.

A peer-reviewed version of this article has been accepted by the Journal of Orthopaedic \& Sports Physical Therapy and is available at https://doi.org/10.2519/jospt.2021.9893. The publisher version of record will become available on April 1, 2021 at the same link.

\section{Keywords Workload, youth, football, soccer, RPE, risk factors}

DOI $10.31236 /$ osf.io/nhqbx

Citation

Dalen-Lorentsen, Andersen, Bjørneboe, Vagle, Martin, Kleppen, Fagerland \& Clarsen (2020) SportRxiv. 


\section{PREPRINT -- NOT PEER REVIEWED}

\section{INTRODUCTION}

The acute-chronic workload ratio (ACWR) was introduced by Hulin and co-workers in 2014[1] as a modification of Banister's fitness-fatigue model [2]. It is calculated by dividing the total amount of training an athlete has recently completed (normally the previous week) by the amount they have completed over a longer time period (e.g., the previous 4 weeks). ACWR is intended to reflect athletes' preparedness for training by accounting for both positive and negative training effects (i.e. fitness and fatigue) [3]. The relationship between ACWR and health problems has emerged as a popular topic among practitioners and researchers alike; since 2014, at least 45 cohort studies and multiple review papers have addressed the topic (supplementary \#1). The quality of the evidence supporting ACWR has, however, been questioned [4], and several methodological aspects of ACWR calculation and analysis have been debated [5-14].

There is no consensus on how the relationship between ACWR and health problems should be calculated and analysed, and no two studies have used the same approach. Although most studies report significant findings (supplementary \#1), their results and conclusions are inconsistent, and few studies consider their negative findings when making conclusions. The inconsistent results among ACWR studies may reflect differences in methodology, particularly (1) how training load and health problems was recorded, (2) how ACWR was calculated, and (3) how the relationship between ACWR and health problems was analysed.
In this study, our aim was to analyse the relationship between ACWR and health problems among elite youth football players using a wide range of methodological combinations defined a priori. We considered the extent to which these methodological choices influenced the relationship between ACWR and health problems.

\section{METHODS}

This study was a prospective cohort study conducted from July to October 2017. During this period, which included 1-2 weeks of summer break and 13-14 weeks of regular in-season competition, players recorded their daily training load and health status using an online survey software (Briteback AB, version 2.5.3.1; Norrköping, Sweden). We obtained approval from the South-Eastern Norway Regional Committee for Medical and Health Research Ethics (2017/1015) and the Norwegian Centre for Research Data (5487) before the study start. All participant players or their guardians provided written informed consent.

\subsection{Recruitment}

We invited a convenience sample of 12 Norwegian teams from the elite U-19 divisions to participate in the study, 6 girls' teams, and 6 boys' teams. Teams that already used a training load monitoring system were excluded $(n=6)$. Six teams ( 3 female and 3 male) accepted the invitation to participate in the study, and all players in these teams agreed to participate. We invited all permanent squad members of these teams, except players with existing health problems that we expected would render them unavailable for foot- 
A cherry tree ripe for picking: The relationship between the acute:chronic workload ratio and health problems

ball training and match play during the study period. We performed no power or sample size calculation before the study.

\subsection{Collection of training load data}

Players reported their daily training load using a short online questionnaire (supplementary file \#2). A link to the questionnaire was distributed by an automated short message service (SMS) at 9 p.m. every evening. If players had not replied to the questionnaire before 8 a.m. the following morning, they received an SMS reminder. The questionnaire included questions with structured response options on training load data for all football activity, including organized training and matches, as well as non-organized football play. Players were asked to record the duration in minutes and their overall perceived rate of physical exertion (RPE) using the modified Borg CR-10 scale [15]. We calculated an arbitrary training load unit (AU) by multiplying the duration and the session RPE (sRPE) [15] for all footballing activity.

\subsection{Collection of health data}

Players recorded their health status in the daily questionnaire by selecting one of three options: (1) no health problem, (2) new health problem, (3) worsening of an existing health problem. Players were asked to report all complaints, irrespective of their consequences on football participation or their need to seek medical attention [16]. If the player reported any new health problems or a worsening of an existing problem, a sports medicine clinician (physiotherapist or chiropractor) contacted them by telephone the following day to conduct a structured interview. During this interview, we classified health problems according to UEFA guidelines as injury or illness, acute or overuse, contact or non-contact, injury mechanism, tissue and body part (supplementary \#3) [17].

\subsection{Efforts to limit potential sources of bias}

We standardized sRPE-registration by collecting data at 9 p.m., when footballing activity was likely to be finished, and when the player was unlikely to be in the presence of their team-mates. To maximize the likelihood of honest reports, we ensured players that all their training and health data would be treated as confidential and not disclosed to their coach or team-mates. We familiarised players with the RPE scale during information meetings before the commencement of the study, and instructed players to consider the whole session/match when giving a RPE score. We contacted coaches weekly to engage their support in motivating their players to respond to the questionnaire, aimed at ensuring high compliance. Any player that consistently failed to respond to the automated reminder-SMS was sent personal messages to encourage them to resume participation in the study. To ensure consistent reporting of all health problems, we familiarized players with the definitions of the prestudy meeting and repeatedly emphasized the importance of reporting all health problems, irrespective of their consequences. 
A cherry tree ripe for picking: The relationship between the acute:chronic workload ratio and health problems

To assess the potential bias in our results, we conducted an anonymous post-study survey of all participants, asking whether the players had reported all training and health data as instructed.

\subsection{Variables}

All independent (training load) and dependent (health status) variables were defined a priori, and we identified sex and age as potential confounders of the association between training load and health status.

\subsubsection{Independent variables}

We calculated ACWR in 8 different ways, using all combinations of the following:

- The "acute" and "chronic" workload periods were defined in two ways: 7 to 28 days and 7 to 21 days.

- Workload was calculated for each period using a simple rolling average (RA) and exponentially weighted moving average (EWMA; for formulas, see supplementary file \#4) [11].

- For RA calculations, we included the acute period in the chronic period (coupled approach) and separated it from the chronic period (uncoupled approach). As the original EWMA formula is coupled by nature[11]. and to limit number of analyses, no uncoupled approach was used.

ACWR variables were then converted into ordinal categorical variables with two methods:
- Pre-defined bins using the most common categories: Low $(<0.8)$, medium (o.8 to 1.5) and high (>1.5)[1, 18].

- Z-scores: Low ( $<1$ standard deviation (SD) below the mean), medium (1 SD below the mean to $1 \mathrm{SD}$ above the mean), high ( $>1$ SD above the mean).

○ Z-score categories for RA was: Low (<0.4), medium (0.4 to 1.81) and high (>1.81)

○ Z-score categories for EWMA was: Low (<0.32), medium (0.32 to 1.73) and high (>1.73)

\subsubsection{Dependent variables}

Using players' daily health reports, we created three different dependent variables for analysis:

- Any worsening in health status (yes/no): This included all new injuries and illnesses, as well as all cases when players reported a worsening of an existing injury or illness

- Any worsening in injury status (yes/no): This included all new injuries and cases when players reported a worsening of an existing injury. Illnesses were excluded from this definition.

- Any new non-contact injury (yes/no)

\subsection{Statistical analyses}

All statistical methods were defined a priori. We used a random-effects logistic regression model to investigate all combinations of the 12 independent and 3 dependent variables, comparing the medium ACWR group to the low and high groups, 
A cherry tree ripe for picking: The relationship between the acute:chronic workload ratio and health problems

and the low group to the high group. We performed a total of 108 (all combinations X comparisons) separate analyses using Stata software (version 15.3 StataCorp LLC, College Station, TX), with the xtlogit command (see supplementary file \#5 for script). A random-intercept model was used, and the random error term was assumed normal distributed (with mean o). We adjusted the regression models for confounding by sex and age, and for the effect of clustering by individuals and teams. In the first round of analyses, we analysed the data unadjusted for sex and age. A sample of 20 analyses was subsequently repeated with adjustment for sex and age. As there were vast differences between results, we chose the adjusted model for our main analyses; however, the unadjusted results are available as a supplementary material (\#5). Incidence was calculated by dividing the total number of cases satisfying each health problem definition by the total exposure time and multiplying by 1000 hours. We calculated the absolute daily risk of health problems by dividing the number of cases satisfying each health problem definition by the total number of athlete days in the study.

\subsection{Missing data}

When athletes' training load data were incomplete, either due to missing session duration or RPE, we replaced the missing values of individual players with the team average for that session. If an entire session was unreported, we did not attempt to estimate missing data. Instead, we defined a minimum amount of information necessary to make an ACWR calculation (5 days for a 7-day acute period, 14 days for a 21-day chronic period and 21 days for a 28 -day chronic period) and used a statistical model (random-effects logistic regression) capable of handling incomplete datasets.

\section{RESULTS}

A total of 86 players agreed to participate, 47 boys (mean age 17.5, SD 0.7), and 39 girls (mean age 17.5, SD 1.3). The overall response to the daily questionnaire was $69 \%$ (6250 of 9030 athlete days). The weekly response varied from $90 \%$ in the first week, to $63 \%$ in the last week.

\subsection{Training load and exposure}

The median weekly training load per player was 1262 AU (interquartile range; IQR 398) for training and $439 \mathrm{AU}$ (IQR 111) for matches. The lowest weekly cumulative training load was in week 1 (832 AU), and the highest cumulative training load was in week 8 (2231 AU). The total exposure during the study, including both training and matches, was 4668 hours. Per player, this corresponded to a median weekly exposure of 242 (IQR 87) training minutes and 64 (IQR 17) match minutes (total: 306 minutes, IQR 32).

\subsection{Health problems}

A total of 196 health problems were reported. Of these, 91 were classified as injuries, 19 as illness, and 88 were not classified. During the study period, a total of 65 players ( $75 \%$ of all players) reported a health problem, 62 players ( $72 \%)$ registered an injury, and 39 (45\%) reported a new 
A cherry tree ripe for picking: The relationship between the acute:chronic workload ratio and health problems

non-contact injury. The incidence and daily risk

of health problems is shown in Table 1.

Table 1 Incidence and absolute daily risk

\begin{tabular}{|l|c|c|c|c|c|}
\hline $\begin{array}{l}\text { Health problem defini- } \\
\text { tion }\end{array}$ & $\mathbf{N}$ & $\begin{array}{c}\text { Incidence per } \\
\mathbf{1 0 0 0 h}\end{array}$ & $\mathbf{9 5 \%}$ CI & $\begin{array}{c}\text { Absolute } \\
\text { daily risk }\end{array}$ & $\mathbf{9 5 \% ~ C I ~}$ \\
\hline All Health problems & 196 & 42.0 & $36.3-48.3$ & $3.14 \%$ & $2.72-3.6$ \\
\hline All Injuries & 91 & 19.4 & $15.6-23.9$ & $1.46 \%$ & $1.17-1.78$ \\
\hline New non-contact injuries & 45 & 9.64 & $0.7-0.13$ & $0.72 \%$ & $0.53-0.96$ \\
\hline
\end{tabular}




\subsection{Main results: Association between ACWR and health problems}

Of the 108 analyses performed, we found 21 (19\%) significant associations between ACWR and health problems, spread across various methodological combinations (figure 1). We did not observe any patterns of combinations that substantially increased the chance of an association. The only option that was not present in any of the associations was moving from medium to low category (table 2). The strongest association we observed was with the following combination: a coupled 7-day acute, 28-day chronic time-period using EWMA, and by using Z-score categories comparing low to high category with all injuries as health problem definition (OR: 11.8, 95\% CI: 1.5-93.2).

Table 2 Number of associations and number of analyses based on variables. (EWMA: Expo-nentially weighted moving average; NNC-Injuries: New non-contact injuries)

\begin{tabular}{|c|c|c|}
\hline & $\mathbf{N}$ of analyses & $\mathbf{N}$ of associations \\
\hline \multicolumn{3}{|l|}{ Average } \\
\hline Rolling average & 72 & $11(15 \%)$ \\
\hline EWMA & 36 & $10(28 \%)$ \\
\hline \multicolumn{3}{|c|}{ Chronic time-period } \\
\hline 28-days & 54 & $10(19 \%)$ \\
\hline 21-days & 54 & $11(20 \%)$ \\
\hline \multicolumn{3}{|l|}{ Coupling } \\
\hline Coupled & 72 & $14(19 \%)$ \\
\hline Non-coupled & 36 & $8(22 \%)$ \\
\hline \multicolumn{3}{|l|}{ Categories } \\
\hline Standard & 54 & $8(15 \%)$ \\
\hline Z-Score & 54 & $13(24 \%)$ \\
\hline \multicolumn{3}{|l|}{ Comparison } \\
\hline Med to low & 36 & $\mathrm{O}(0 \%)$ \\
\hline Med to high & 36 & $10(28 \%)$ \\
\hline Low to high & 36 & $11(31 \%)$ \\
\hline \multicolumn{3}{|c|}{ Health problem definition } \\
\hline All health problems & 36 & $12(33 \%)$ \\
\hline All injuries & 36 & $9(25 \%)$ \\
\hline NNC-Injuries & 36 & $\mathrm{O}(0 \%)$ \\
\hline
\end{tabular}


A cherry tree ripe for picking: The relationship between the acute:chronic workload ratio and health problems
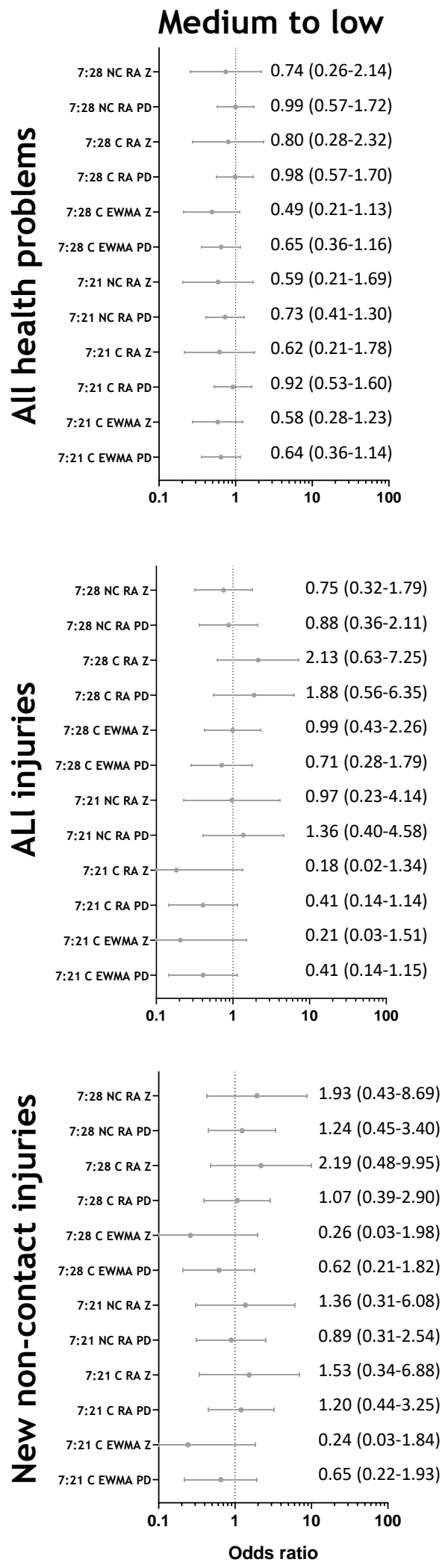

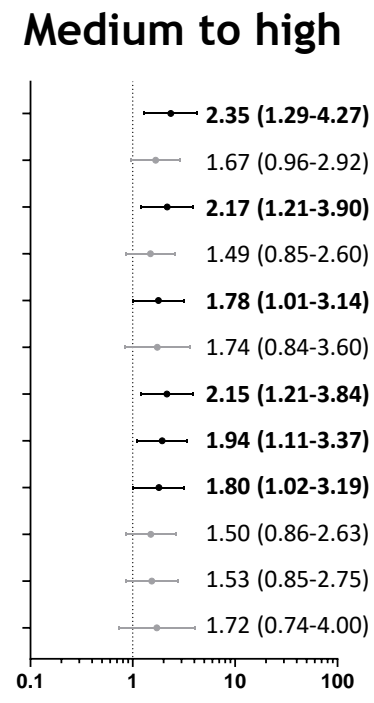

\section{Low to high}
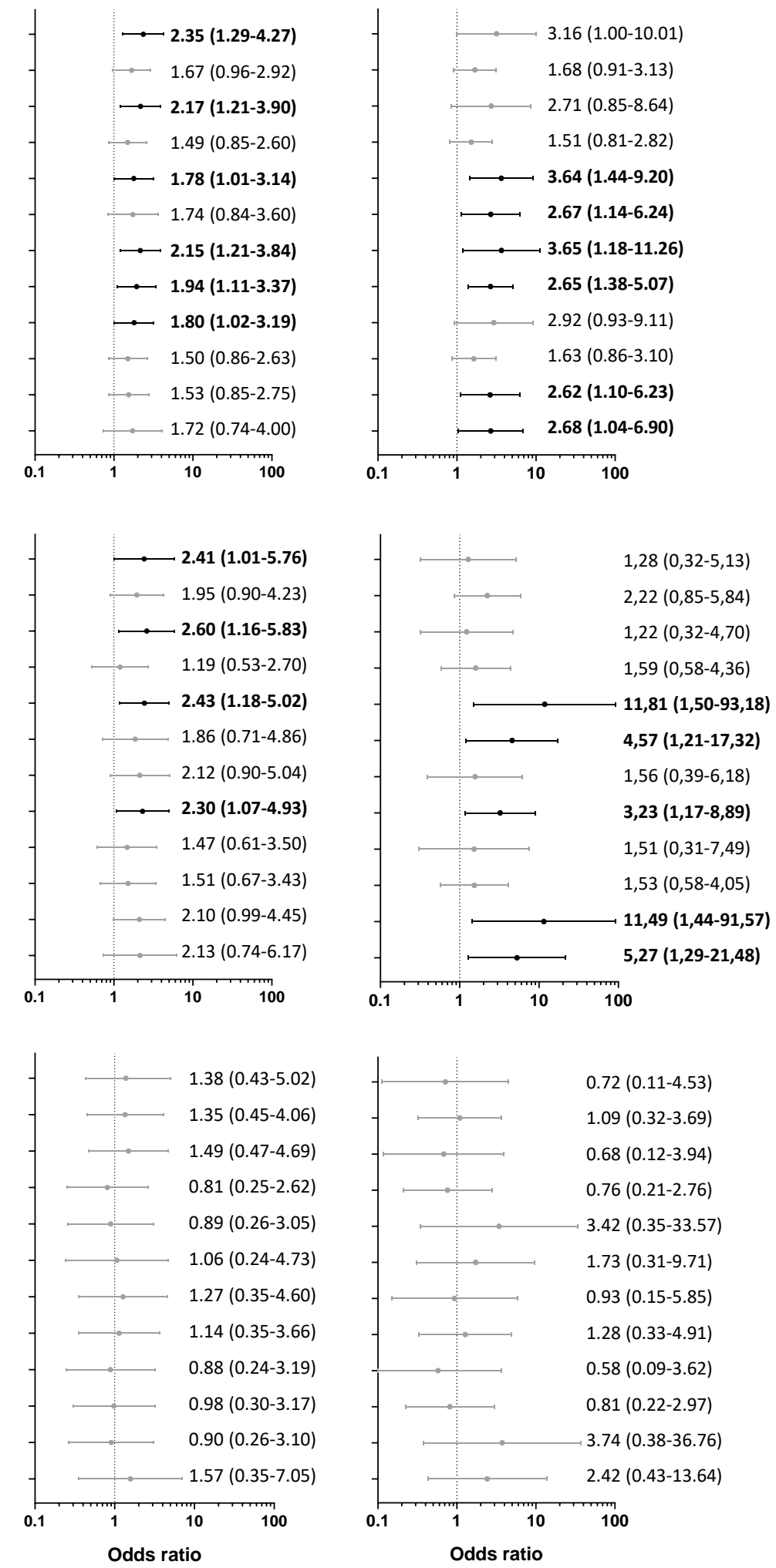

Figure 1 1Relationship between ACWR and all health problems, all injuries and new non-contact injuries. C=Coupled, NC=Non-coupled, RA= Rolling average, EWMA = Exponentially weighted moving average, $Z=Z$-score based categories, $P D=P r e$-defined categories. For all details, see supplementary material \#5 
A cherry tree ripe for picking: The relationship between the acute:chronic workload ratio and health problems

\subsection{Post-study survey}

Forty-five players (53\%) responded to the poststudy survey. Of these, 15 (33\%) players said they had not reported training load data every day as instructed. Participants described the two main reasons why they did not report training load data: (1) they had forgotten to reply to the SMS (9 players), and (2) that it was too time-consuming (2 players). Thirteen players (29\%) admitted they had not reported all health problems, with the most frequent explanations that they did not think the problem was significant enough (8 players) and that they did not want to be contacted by phone for the follow-up interview (3 players).

\section{DISCUSSION}

This study aimed to explore the effect on different methodological choices on the association between ACWR and health problems. As this was a critique of current methods in ACWR research, we based our choice of methods on the published literature and do not necessarily endorse their future use. We calculated ACWR in 12 different ways, used three different combinations, and used three different outcome definitions, resulting in a total of 108 separate analyses. Of these, 21 (19\%) identified an association between ACWR and health problems. There was a large variation in the size and precision of the estimated strength of the association, and we were unable to identify strong patterns in the results in favour of one particular methodological approach. Our main conclusion is that the potential association between
ACWR and health problems is highly affected by methodological choices.

\subsection{Health problem definition}

The definition of a health problem had the largest effect on the number of associations identified between ACWR and health problems. When we applied the broadest definition, which included all injuries and illnesses, one in three analyses yielded an association. When this definition was limited to injuries and not illnesses, one in four analyses yielded an association. However, when we defined the outcome as "new non-contact injuries", we did not identify any associations. One likely explanation for this finding is that the number of cases was reduced from 196 to 45 when we applied a narrower health problem definition. As demonstrated by Bahr and Holme,[19] this is likely to be too few cases to detect a small to moderate association (which requires approximately 200 cases) [19]. As the median number of cases in similar studies is 72 (supplementary \#1), a large proportion of studies in this field appear to be underpowered.

Most commonly, ACWR studies have used a timeloss health problems definition [1, 20-49]. However, this narrow definition is unlikely to capture a majority of overuse injuries, which do not often lead to time loss [50-52]. As overuse injuries are likely the injury type most associated with training load, we believe it is essential to use a broad health problems definition in future training load studies. However, this requires an appropriate study design $[52,53]$. 
A cherry tree ripe for picking: The relationship between the acute:chronic workload ratio and health problems

\subsection{ACWR calculation}

The duration of the acute and chronic time-periods varies considerably between the previous ACWR studies. Time-periods are often sport-specific and typically aim to represent one training micro-cycle (acute) and one macro-cycle (chronic) [54]. We chose the two most commonly used chronic periods, 28 days and 21 days and found no obvious differences between the two. Most studies define the acute period as 7 days (supplementary \#1); however, some define it differently (e.g. 3 day) $[54,55]$. We did not alter the duration of the acute period, largely to limit the number of analyses in this study.

Most commonly, the average acute and chronic workloads are determined using a simple rolling average of the entire time period $[1,18,20,21,23-$ 28, 30-34, 38-41, 43-49, 56-65]. However, Williams et al.[11] proposed the use of an EWMA, whereby each day's contribution to the aggregate is exponentially greater than the previous day. Several studies have shown EWMA-based ACWR calculations to be more closely associated to health problems than RA-based calculations [18, $26,38,42]$. Our findings support this, with 35 of the 36 highest odds ratios obtained using EWMA. However, we emphasise that more than twothirds of our analyses using EWMA did not identify any association between ACWR and health problems.

Recently, Lolli et al.[12, 13] criticized the use of coupled ACWR, as it by definition leads to a correlation between the numerator and the denominator in the ratio. Our results suggest that coupling is not the most important methodological choice, as we identified a similar proportion of associations using a coupled and uncoupled approach. Nevertheless, we highlight that ACWR values calculated using each approach cannot be directly compared.

\subsection{ACWR analysis}

Almost all training load data are converted from a continuous to a categorical variable for analysis. Recently, Carey et al. highlighted problems with large false discovery and rejection rates with this approach [5]. Loss of power and residual confounding are other important problems with discretization of continuous variables [66, 67], and cannot be recommended in ACWR research. Despite the challenges, few attempts have been made to investigate ACWR as a continuous variable [31] . Instead, studies have categorised ACWR values according to the first ACWR publication [1], or by z scores (typically \pm 1 ). We categorised data in two ways, using pre-defined cut-off values of 0.8 and 1.5 and using a z-score of \pm 1 , and there was not a large difference between the two ( 8 and 13 significant results, respectively). Rather, the limitations of each approach should be recognised. If ACWR data are normally distributed, categorising data using a z-score of 1.5 would mean that approximately $7 \%$ of values would be classified as "high" and $7 \%$ as "low". In small sample sizes, or in any sample size that discretises into small brackets, sparse data is likely to effect results $[14,68]$. Furthermore, this approach provides a relative categorisation, such that in a group of athletes whose training loads fluctuate greatly, only extreme values would be classified as 
A cherry tree ripe for picking: The relationship between the acute:chronic workload ratio and health problems

low or high. However, the most commonly used categories were based on studies of elite rugby league and cricket players [1, 47], and are unlikely to be universally applicable across all sporting contexts [5].

After choosing discretizing approach, authors then must choose number of categories and which categories that are compared. Both number of categories and comparisons are widely varied across the literature (supplementary \#1). We calculated the odds of sustaining health problems between days with low and high, medium and high, and medium and low ACWR. In both cases involving an increased ACWR (i.e. medium to high and low to high) we found significantly higher odds of developing a health problem in approximately $1 / 3$ of analyses. In contrast, we did not identify any associations when comparing medium to low ACWR. This finding contrasts with the originallydescribed ACWR concept, which described an increased injury risk when ACWR was low [3]. We note that few $(n=2)[43,65]$ other studies that have supported this aspect of the concept and question its biological rationale.

We believe that discretisation is inappropriate in ACWR-injury studies and a method for modelling training load as a continuous variable is warranted.

\subsection{Statistical approach}

A variety of statistical models have been used to analyse the workload between ACWR and health problems. According to a recent review [70], most studies have used an overly-simple approach, without accounting for the multifactorial aetiology of injuries, between-athlete or within-athlete assessments, missing and unbalanced data, and the nature of time-varying variables [70]. To tackle some of these challenges, we chose a random-effects logistic regression model, which is one of two recommended approaches [70]. Nevertheless, we believe that research comparing the results of different statistical approaches is warranted.

\subsection{Methodological considerations}

In this study we collected health data directly from athletes; a design which has not previously been used in ACWR studies. This approach allowed us to use a broad definition of health problem, which means we are likely to have captured a far greater proportion of overuse injuries than previous studies [52, 53]. However, this approach also has limitations. Health problems were not medically confirmed, lacked diagnostic details, and in many cases were not fully classified (e.g. as acute or overuse injuries). Furthermore, players failed to respond to $31 \%$ of the daily questionnaires and, as illustrated by our post-study survey, did not always report every health problem or training session, potentially leading to selection bias. However, there are also limitations with collecting data from medical or coaching staff [ 71 , 72]. Therefore, in future studies we believe that data should be collected using a variety of different sources (e.g. athlete reports, attendance logs, medical records, etc.).

Session-RPE is considered a validated method for measuring training load across various sports 
A cherry tree ripe for picking: The relationship between the acute:chronic workload ratio and health problems

[73], and for elite youth footballers specifically [74]. Players reported at 9 PM in our study, giving a potential of 12 hours delay after a session is finished before reporting the sRPE results. Even though the general recommendation is to collect sRPE 30-minutes post-session [15], a recent study investigating the potential recall bias by reporting late, found no difference when SRPE was reported 48-hours post-match compared to directly after the match [75]. We chose sRPE because it was the most practical way to quantify load in footballers of this level, and because a large majority of previous ACWR studies have used it as their primary load measure [18, 23, 25-28, 31, 32, 34, 36, 39, $40,43,44,48,49,55-57,62,65,76]$. It is unknown whether our results would have been different if we had used another method to quantify load (e.g.. microsensors).

Handling of missing data might affect our results, and we used a sub-optimal method where the team average replaced missing values when either duration or RPE were missing. Although this is the most common method used in previous studies, future research should investigate better options to handle missing data in training load research. We cannot exclude the possibility that discretization of ACWR has influenced results through loss of data, false discovery and rejection rates, residual confounding and sparse data bias [68].

\subsection{Future directions}

All previous ACWR studies have used different methodological approaches and their findings are inconsistent. In general, the conclusions of these studies have focussed on the significant findings. In this study, we have demonstrated the number of non-significant findings likely to be behind every positive one. Reporting bias is therefore an important consideration when assessing the overall evidence for the relationship between ACWR and health problems.

In exploratory research, performing many analyses is often justified. However, in future ACWR studies, we encourage researchers to report all analyses they perform, not just their significant findings. As for randomised controlled trials, protocol pre-registration should be considered. Although each sport and study population are different, methodological consensus for workload studies is needed. Unfortunately, we believe that the extent and quality of the evidence is currently insufficient to inform any consensus process.

Finally, we question the need for more underpowered risk factor studies examining the relationship between training load and health problems. We believe that to improve our understanding of the relationship between ACWR and health problems; it is necessary to conduct much larger observational studies (i.e. >1000 injuries), as well as experimental research such as randomised controlled trials. 
A cherry tree ripe for picking: The relationship between the acute:chronic workload ratio and health problems

\section{Acknowledgments}

The Oslo Sports Trauma Research Center has been established at the Norwegian School of Sport Sciences through generous grants from the Royal Norwegian Ministry of Culture, the South-Eastern Norway Regional Health Authority, the International Olympic Committee, the Norwegian Olympic Committee and Confederation of Sport, and Norsk Tipping AS. The authors are grateful to the assessors, and to all the players and coaches who participated in the study.

The authors would like to acknowledge Adam Sullivan in helping us with initial EWMA calculations and Eirik Halvorsen Wik for valuable feedback on the draft manuscript.

\section{Funding}

The Oslo Sports Trauma Research Center has been established at the Norwegian School of Sport Sciences through generous grants from the Royal Norwegian Ministry of Culture, the South-Eastern Norway Regional Health Authority, the International Olympic Committee, the Norwegian Olympic Committee and Confederation of Sport, and Norsk Tipping AS

\section{Conflict of interest /Competing Interests}

None

\section{Availability of data and material}

All raw data are available in supplementary files

\section{Code availability}

All code is available in supplementary files

\section{Ethics approval}

We obtained approval from the South-Eastern Norway Regional Committee for Medical and
Health Research Ethics (2017/1015) and the Norwegian Centre for Research Data (5487) before the study start. All participant players or their guardians provided written informed consent.

\section{Consent to participate}

All participant players or their guardians provided written informed consent to participate.

\section{Consent for publication}

All participant players or their guardians provided written informed consent for publication.

\section{Authors contribution}

TD, BC, TEA, JB, and MWF planned the study. The data collection was done by TD, KNM, MV, and MK. All authors have been involved in the data analyses, drafting, and revision of the manuscript, and all have approved the final version.

\section{REFERENCES}

1. Hulin BT, Gabbett HT, Blanch P, Chapman P, Bailey D, Orchard JW. Spikes in acute workload are associated with increased injury risk in elite cricket fast bowlers. British journal of sports medicine. 2014 Mar 23;48(8):708-12.

2. Banister EW, Calvert TW, Savage MV, Bach T. A systems model of training for athletic performance: Aust J Sports Med; 1975.

3. Gabbett TJ. The training-injury prevention paradox: should athletes be training smarter and harder? Br J Sports Med. 2016 Mar;50(5):273-80.

4. Impellizzeri FM, Woodcock S, McCall A, Ward $P$, Coutts AJ. The acute-chronic 
A cherry tree ripe for picking: The relationship between the acute:chronic workload ratio and health problems

workload ratio-injury figure and its 'sweet spot' are flawed. 2019 2019, June 03.

5. Carey DL, Crossley KM, Whiteley R, Mosler A, Ong KL, Crow J, et al. Modeling Training Loads and Injuries: The Dangers of Discretization. Med Sci Sports Exerc. 2018 Nov;50(11):2267-76.

6. Sampson JA, Fullagar HH, Murray A. Evidence is needed to determine if there is a better way to determine the acute:chronic workload. Br J Sports Med. 2017 Apr;51(7):621-2.

7. Menaspà P. Are rolling averages a good way to assess training load for injury prevention? British journal of sports medicine. 2016 May 24:bjsports-2016096131.

8. Drew MK, Blanch P, Purdam C, Gabbett TJ. Yes, rolling averages are a good way to assess training load for injury prevention. Is there a better way? Probably, but we have not seen the evidence. Br J Sports Med. 2017 Apr;51(7):618-9.

9. Windt J, Gabbett HT. Is it all for naught? What does mathematical coupling mean for acute:chronic workload ratios?; 2018.

10. Hulin BT, Gabbett HT. Indeed association does not equal prediction: the neverending search for the perfect acute:chronic workload ratio. British journal of sports medicine. 2018 Jun 09:bjsports-2018-099448-2.

11. Williams S, West $S$, Cross MJ, Stokes KA. Better way to determine the acute:chronic workload ratio? British journal of sports medicine. 2016 Sep 20:bjsports-2016-096589-3.
12. Lolli L, Batterham AM, Hawkins R, Kelly DM, Strudwick AJ, Thorpe R, et al. Mathematical coupling causes spurious correlation within the conventional acuteto-chronic workload ratio calculations. British Journal of Sports Medicine. 2019;53(15):921.

13. Lolli L, Batterham AM, Hawkins R, Kelly DM, Strudwick AJ, Thorpe RT, et al. The acute-to-chronic workload ratio: an inaccurate scaling index for an unnecessary normalisation process? British Journal of Sports Medicine. 2018:bjsports-2017-098884.

14. Wang C, Trejo Vargas J, Stokes T, Steele R, Shrier I. The acute:chronic workload ratio: challenges and prospects for improvement. arXiv e-prints; 2019.

15. Foster C, Florhaug JA, Franklin J, Gottschall L, Hrovatin LA, Parker S, et al. A New Approach to Monitoring Exercise Training. Journal of Strength and Conditioning Research. 2001;15(1):10915.

16. Fuller CW, Ekstrand J, Junge A, Andersen TE, Bahr R, Dvorak J, et al. Consensus statement on injury definitions and data collection procedures in studies of football (soccer) injuries. Br J Sports Med. 2006 Mar;40(3):193-201.

17. Hägglund $M$, Waldén $M, B a h r ~ R$, Ekstrand J. Methods for epidemiological study of injuries to professional football players: developing the UEFA model. British Journal of Sports Medicine. 2005;39(6):340.

18. Johnston R, Cahalan R, Bonnett $\mathrm{L}$, Maguire $M$, Nevill A, Glasgow $P$, et al. Training Load 
A cherry tree ripe for picking: The relationship between the acute:chronic workload ratio and health problems

and Baseline Characteristics Associated

With New Injury/Pain Within an

Endurance Sporting Population: A

Prospective Study. International Journal of Sports Physiology and Performance.

2019;14(5):590-7.

19. Bahr R, Holme I. Risk factors for sports injuries - a methodological approach. British Journal of Sports Medicine. 2003;37(5):384.

20. Bowen L, Gross AS, Gimpel M, Li FX. Accumulated workloads and the acute:chronic workload ratio relate to injury risk in elite youth football players. Br J Sports Med. 2017 Mar;51(5):452-9.

21. Bowen L, Gross AS, Gimpel M, Bruce-Low $S$, Li F-X. Spikes in acute:chronic workload ratio (ACWR) associated with a 5-7 times greater injury rate in English Premier League football players: a comprehensive 3-year study. British journal of sports medicine. 2019 Feb 21:bjsports-2018099422-9.

22. Colby MJ, Dawson B, Heasman J, Rogalski B, Gabbett HT. Accelerometer and GPSderived running loads and injury risk in elite Australian footballers. Journal of strength and conditioning research / National Strength \&amp; Conditioning Association. 2014 Aug;28(8):2244-52.

23. Cross MJ, Williams S, Trewartha G, Kemp SP, Stokes KA. The Influence of In-Season Training Loads on Injury Risk in Professional Rugby Union. Int J Sports Physiol Perform. 2016 Apr;11(3):350-5.

24. Cummins C, Welch M, Inkster B, Cupples $B$, Weaving $D$, Jones $B$, et al. Modelling the relationships between volume, intensity and injury-risk in professional rugby league players. Journal of Science and Medicine in Sport. 2019 Jan 12:1-8.

25. Delecroix B, McCall A. Workload and noncontact injury incidence in elite football players competing in European leagues. Taylor \&amp; Francis. 2019.

26. Esmaeili A, Hopkins WG, Stewart AM, Elias GP, Lazarus BH, Aughey RJ. The Individual and Combined Effects of Multiple Factors on the Risk of Soft Tissue Non-contact Injuries in Elite Team Sport Athletes. Frontiers in Physiology. 2018 2018September-21;9(1280).

27. Fanchini $M$, Rampinini E, Riggio $M$, Coutts AJ, Pecci C, McCall A. Despite association, the acute:chronic work load ratio does not predict non-contact injury in elite footballers. Science and Medicine in Football. 2018 2018/04/03;2(2):108-14.

28. Harrison PW, Johnston RD. Relationship Between Training Load, Fitness, and Injury Over an Australian Rules Football Preseason. The Journal of Strength \& Conditioning Research. 2017;31(10):268693.

29. Jaspers A, Kuyvenhoven JP, Staes F, Frencken WGP, Helsen WF, Brink MS. Examination of the external and internal load indicators' association with overuse injuries in professional soccer players. Journal of Science and Medicine in Sport. 2017 Oct 24:1-7.

30. Kountouris A, Sims K, Beakley D, Saw AE, Orchard J, Rotstein A, et al. MRI bone marrow oedema precedes lumbar bone stress injury diagnosis in junior elite 
A cherry tree ripe for picking: The relationship between the acute:chronic workload ratio and health problems

cricket fast bowlers. British Journal of

Sports Medicine. 2019;53(19):1236.

31. Lathlean T, Gastin P, Newstead S, Finch

CF. Absolute and Relative Load and Injury in Elite Junior Australian Football Players

Over 1 Season. International Journal of Sports Physiology and Performance.

2019:1-9.

32. Lu D, Howle K, Waterson A, Duncan C, Duffield R. Workload profiles prior to injury in professional soccer players. Science and Medicine in Football. 2017 2017/09/02;1(3):237-43.

33. Malone S, Hughes B, Doran DA, Collins K, Gabbett HT. Can the workload-injury relationship be moderated by improved strength, speed and repeated-sprint qualities? Journal of Science and Medicine in Sport. 2018 Feb 01:1-17.

34. Malone S, Owen A, Newton M, Mendes B, Collins KD, Gabbett TJ. The acute:chonic workload ratio in relation to injury risk in professional soccer. J Sci Med Sport. 2017 Jun;20(6):561-5.

35. Malone S, Owen AL, Mendes B, Hughes B, Collins K, Gabbett HT. High-speed running and sprinting as an injury risk factor in soccer: Can well-developed physical qualities reduce the risk? Journal of Science and Medicine in Sport. 2017 May 24:1-21.

36. McCall A, Dupont G, Ekstrand J. Internal workload and non-contact injury: a oneseason study of five teams from the UEFA Elite Club Injury Study. Br J Sports Med. 2018 Dec;52(23):1517-22.
37. Murray NB, Gabbett HT, Townshend AD, Hulin BT, McLellan CP. Individual and combined effects of acute and chronic running loads on injury risk in elite Australian footballers. Scandinavian Journal of Medicine \&amp; Science in Sports. 2016 Jul 15:1-9.

38. Murray NB, Gabbett TJ, Townshend AD, Blanch P. Calculating acute:chronic workload ratios using exponentially weighted moving averages provides a more sensitive indicator of injury likelihood than rolling averages. $\mathrm{Br} \mathrm{J}$ Sports Med. 2017 May;51(9):749-54.

39. Pote L, Christie CJ. Workloads placed on adolescent cricket players: A pilot study. International Journal of Sports Science \& Coaching. 2019 2019/02/01;14(1):107-13.

40. Raya-González j, Nakamura F, Castillo F, Yanci J, Fanchini M. Determining the Relationship Between Internal Load Markers and Noncontact Injuries in Young Elite Soccer Players. International Journal of Sports Physiology and Performance. 2019;14(4):421-5.

41. Sampson JA, Murray A, strength SWJo, 2019. Subjective Wellness, Acute: Chronic Workloads, and Injury Risk in College Football. europepmcorg. 2019 Feb:1.

42. Sampson JA, Murray A, Williams S, Halseth $T$, Hanisch J, Golden G, et al. Injury riskworkload associations in NCAA American college football. Journal of Science and Medicine in Sport. 2018 2018/12/01/;21(12):1215-20.

43. Stares J, Dawson B, Peeling P, Heasman J, Rogalski $B$, Drew $M$, et al. Identifying high risk loading conditions for in-season injury 
A cherry tree ripe for picking: The relationship between the acute:chronic workload ratio and health problems

in elite Australian football players. J Sci

Med Sport. 2018 Jan;21(1):46-51.

44. Thornton HR, Delaney JA, Duthie GM,

Dascombe BJ. Importance of Various

Training-Load Measures in Injury

Incidence of Professional Rugby League

Athletes. International Journal of Sports

Physiology and Performance. 2017

Jul;12(6):819-24.

45. Warren A, Williams S, McCaig S,

Trewartha G. High acute:chronic workloads are associated with injury in England \& Wales Cricket Board

Development Programme fast bowlers. Journal of Science and Medicine in Sport. 2018 2018/01/01/;21(1):40-5.

46. Windt J, Gabbett HT, Ferris D, Khan KM. Training load--injury paradox: is greater preseason participation associated with lower in-season injury risk in elite rugby league players? British journal of sports medicine. 2017 Apr;51(8):645-50.

47. Hulin BT, Gabbett HT, Lawson DW, Caputi $P$, Sampson JA. The acute:chronic workload ratio predicts injury: high chronic workload may decrease injury risk in elite rugby league players. British journal of sports medicine. 2016 Feb;50(4):231-6.

48. Watson A, Brickson S, Brooks A, Dunn W. Subjective well-being and training load predict in-season injury and illness risk in female youth soccer players. British journal of sports medicine. $2016 \mathrm{Dec}$ 05:bjsports-2016-096584.

49. Carey DL, Blanch P, Ong K-L, Crossley KM, Crow J, Morris ME. Training loads and injury risk in Australian football-differing acute: chronic workload ratios influence match injury risk. British journal of sports medicine. 2016 Oct 26:bjsports-2016096309.

50. Clarsen B, Ronsen O, Myklebust G, Florenes TW, Bahr R. The Oslo Sports Trauma Research Center questionnaire on health problems: a new approach to prospective monitoring of illness and injury in elite athletes. Br J Sports Med. 2014 May;48(9):754-60.

51. Bahr R. No injuries, but plenty of pain? On the methodology for recording overuse symptoms in sports. British Journal of Sports Medicine. 2009;43(13):966.

52. Clarsen B, Bahr R. Matching the choice of injury/illness definition to study setting, purpose and design: one size does not fit all! British Journal of Sports Medicine. 2014;48(7):510.

53. Clarsen B. Current severity measures are insufficient for overuse injuries. Science and Medicine in Football. 2016;1(1):91-2.

54. Carey DL, Blanch P, Ong KL, Crossley KM, Crow J, Morris ME. Training loads and injury risk in Australian football-differing acute: chronic workload ratios influence match injury risk. Br J Sports Med. 2017 Aug;51(16):1215-20.

55. Ahmun R, McCaig S, Tallent J, Williams S, Gabbett T. Association of Daily Workload, Wellness, and Injury and Illness During Tours in International Cricketers. International Journal of Sports Physiology and Performance.14(3):369-77.

56. Colby MJ, Dawson B, Peeling P, Heasman J, Rogalski B, Drew MK, et al. Multivariate 
A cherry tree ripe for picking: The relationship between the acute:chronic workload ratio and health problems

modelling of subjective and objective monitoring data improve the detection of non-contact injury risk in elite Australian footballers. Journal of Science and Medicine in Sport. 2017 2017/12/01/;20(12):1068-74.

57. Jaspers A, Brink MS, Probst SGM, Frencken WGP, Helsen WF. Relationships Between Training Load Indicators and Training Outcomes in Professional Soccer. Sports Medicine. 2016 Jul 26:1-12.

58. Hulin BT, Gabbett HT, Pickworth NJ, Johnston R, Jenkins DJ. Relationships Among PlayerLoad, High-Intensity Intermittent Running Ability, and Injury Risk in Professional Rugby League Players. International Journal of Sports Physiology and Performance. 2019:1-7.

59. Li RT, Salata MJ, Rambhia S, Sheehan J, Voos JE. Does Overexertion Correlate With Increased Injury? The Relationship Between Player Workload and Soft Tissue Injury in Professional American Football Players Using Wearable Technology. Sports Health. 2019:1941738119868477.

60. Mehta S. Relationship between workload and throwing injury in varsity baseball players. Physical Therapy in Sport. 2019 2019/11/01/;40:66-70.

61. Murray NB, Gabbett HT, Townshend AD. Relationship Between Pre-Season Training Load and In-Season Availability in Elite Australian Football Players. International Journal of Sports Physiology and Performance. 2016 Nov 11:1-21.

62. O'Keeffe S, O'Connor S, Ní Chéilleachair N. Are internal load measures associated with injuries in male adolescent Gaelic football players? European Journal of Sport Science. 2019:1-12.

63. Ruddy JD, Pollard CW, Timmins RG, Williams MD, Shield AJ, Opar DA. Running exposure is associated with the risk of hamstring strain injury in elite Australian footballers. British Journal of Sports Medicine. 2018;52(14):919.

64. Timoteo TF, Debien PB, Miloski B, Werneck FZ, Gabbett T, Bara Filho MG. Influence of Workload and Recovery on Injuries in Elite Male Volleyball Players. The Journal of Strength \& Conditioning Research. 2018; Publish Ahead of Print.

65. Weiss KJ, Allen SV, McGuigan MR, Whatman CS. The Relationship Between Training Load and Injury in Men's Professional Basketball Players. International Journal of Sports Physiology and Performance. 2017 Mar 02:1-20.

66. Bennette $C$, Vickers A. Against quantiles: categorization of continuous variables in epidemiologic research, and its discontents. BMC Medical Research Methodology. 2012 2012/02/29;12(1):21.

67. Altman DG, Royston P. The cost of dichotomising continuous variables. BMJ. 2006;332(7549):1080.

68. Greenland S, Mansournia MA, Altman DG. Sparse data bias: a problem hiding in plain sight. BMJ. 2016;352:i1981.

69. Hulin BT, Gabbett HT, Caputi P, Lawson DW, Sampson JA. Low chronic workload and the acute:chronic workload ratio are more predictive of injury than betweenmatch recovery time: a two-season prospective cohort study in elite rugby 
A cherry tree ripe for picking: The relationship between the acute:chronic workload ratio and health problems

league players. British journal of sports medicine. 2016 Feb 05;50(16):bjsports2015-095364-1012.

70. Windt J, Ardern CL, Gabbett HT, Khan KM, Cook CE, Sporer BC, et al. Getting the most out of intensive longitudinal data: a methodological review of workload-injury studies. BMJ open. 2018 Oct 02;8(10):e022626-17.

71. Wik EH, Materne O, Chamari K, Duque JDP, Horobeanu C, Salcinovic B, et al. Involving research-invested clinicians in data collection affects injury incidence in youth football. Scandinavian Journal of Medicine \& Science in Sports.

2019;29(7):1031-9.

72. Bjørneboe J, Flørenes TW, Bahr R, Andersen TE. Injury surveillance in male professional football; is medical staff reporting complete and accurate? Scandinavian Journal of Medicine \& Science in Sports. 2011 2011/10/01;21(5):713-20.

73. Haddad M, Stylianides G, Djaoui L, Dellal A, Chamari K. Session-RPE Method for Training Load Monitoring: Validity, Ecological Usefulness, and Influencing Factors. Frontiers in Neuroscience. 2017 2017-November-02;11(612).

74. Impellizzeri FM, Rampinini E, Coutts AJ. Use of RPE-based training load in soccer. Medicine and science .... 2004.

75. Fanchini M, Ferraresi I, Petruolo A, Azzalin $A$, Ghielmetti $R$, Schena $F$, et al. Is a retrospective RPE appropriate in soccer? Response shift and recall bias. Science and Medicine in Football. 2016;1(1):53-9.
76. Malone S, Roe M, Doran DA, Gabbett TJ, Collins KD. Protection Against Spikes in Workload With Aerobic Fitness and Playing Experience: The Role of the Acute:Chronic Workload Ratio on Injury Risk in Elite Gaelic Football. Int J Sports Physiol Perform. 2017 Mar;12(3):393401 